\title{
A crest factor reduction scheme based on recursive optimum frequency domain matrix
}

\begin{abstract}
One of the main imperfections in orthogonal frequency division multiplexing (OFDM) techniques is a peak-to-average power ratio (PAPR) or crest factor (CF). High crest factor signal forces a power amplifier in communication systems to back-off which leads to power efficiency degradation. Several techniques have been proposed in literature to mitigate the high peaks in OFDM signal; however most of them suffer from complexity and PAPR performance. In conventional SLM (C-SLM), increasing the number of inverse fast Fourier transform (IFFT) leads to excessive hardware resources requirement and increases system cost. In this paper a novel crest factor scheme based on recursive optimum frequency domain matrix is proposed which requires only two IFFT which results in significant reduction in the computational complexity. Simulation results show both the complexity and crest factor are reduced significantly compared to the conventional methods.
\end{abstract}

Keyword: Orthogonal frequency division multiplexing; Peak-to-average power ratio; Crest factor; Power amplifier 